\title{
HORMIGAS (Hymenoptera: Formicidae) PRESENTES EN EL CUCBA, ZAPOPAN, JALISCO, MÉXICO
}

\author{
Ants (Hymenoptera: Formicidae) present in CUCBA, Zapopan, Jalisco, Mexico
}

Miguel Vásquez-Bolaños

Entomología, Centro de Estudios en Zoología, Centro Universitario de Ciencias Biológicas y Agropecuarias, Universidad de Guadalajara. Ramón Padilla Sánchez No. 2100, Las Agujas, Zapopan, Jalisco, México C. P. 45220.

Autor para correspondencia: mvb14145@ hotmail.com

\section{Resumen}

Los ambientes alterados por el hombre representan una oportunidad para algunas especies, sobre todo para las especies introducidas e invasoras, tal es el caso de las hormigas. Para conocer la cantidad de especies de hormigas en el Centro Universitario de Ciencias Biológicas y Agropecuarias en Zapopan, Jalisco, México, se colectaron hormigas de manera directa entre 2011 y 2020, el material está depositado en la Colección Entomológica del Centro de Estudios en Zoología de la Universidad de Guadalajara. Se elaboró una lista de las especies colectadas. Para el área del CUCBA se encontraron 39 especies, 22 géneros y seis subfamilias de hormigas. Se tienen nueve especies introducidas: Anoplolepis gracilipes, Cardiocondyla minutior, Paratrechina longicornis y Tapinoma melanocephalum de Asia; Cardiocondyla emeryi, Hypoponera punctatissima, Monomorium pharaonis y Tetramorium simillimum de África; Linepithema humile, de América del Sur. En zonas fragmentadas, también llamadas ecotono o de borde, se puede encontrar una alta riqueza de especies de hormigas, tanto las nativas como las introducidas e invasoras que se ven favorecidas con el nuevo ambiente. No se conoce el impacto que causan las especies introducidas hacia las especies nativas.

Palabras clave: Fragmentación, especies introducidas, mirmecofauna urbana.

\begin{abstract}
The environments disturbed by human represent an opportunity for some species, especially for the introduced and invasive species, such is the case of ants. To know the number of species of ants at the University Center for Biological and Agricultural Sciences in Zapopan, Jalisco, Mexico, it was collected directly between 2011 and 2020, the material is deposited in the Entomological Collection of the Center for Studies in Zoology of the University of Guadalajara. A list of the collected species was prepared. For the CUCBA area, 39 species, 22 genera and six subfamilies of ants were found. There are nine introduced species: Anoplolepis gracilipes, Cardiocondyla minutior, Paratrechina longicornis and Tapinoma melanocephalum from Asia; Cardiocondyla emeryi, Hypoponera punctatissima, Monomorium pharaonis and Tetramorium simillimum from Africa; Linepithema humile, from South America. In fragmented areas, also called ecotone or edge, is posible find a high
\end{abstract}


richness of ant species, native, introduced and invasive, which are favored by the new environment. The impact of introduced species over native species is unknown.

Key words: Fragmentation, introduced species, urban myrmecofauna.

\section{Introducción}

Las hormigas se pueden usar como organismos bioindicadores debido a su alta diversidad, gran abundancia, variedad de funciones en el ecosistema, respuesta rápida a cambios ambientales, fácil muestreo y situación taxonómica relativamente resuelta; esto los convierte en organismos clave para el entendimiento y la conservación de ecosistemas (Arcila y Lozano-Zambrano, 2003). Muchas especies de hormigas se ven favorecidas por la alteración de ambientes naturales y son capaces de habitar en áreas alteradas, desde zonas urbanas hasta agroecositemas, hay especies de hormigas que toleran las actividades humanas sobre todo aquellas denominadas introducidas e invasoras que en la mayoría de los casos se les consideradas plaga (Chacón de Ulloa, 2003). Se conocen más 15,000 especies de hormigas a nivel mundial; para México se tienen poco más de 1,000 especies y 120 para Jalisco; pero esta cifra podría ser mucho mayor debido a la complejidad de ambientes debido a la confluencia entre las regiones Neártica y Neotropical, confiriéndole a México una gran riqueza específica y ser considerado un país megadiverso, con alrededor del $10 \%$ de la diversidad a nivel mundial y un alto número de endemismos (VásquezBolaños, 2015; Dáttilo et al., 2020). Sus hábitos alimentarios, generalistas en la mayoría de los casos, les permiten adaptarse y establecerse en aquellos ambientes que presentan cambio en el uso de suelo provocado por el hombre, desde deforestación hasta incendios con fines agrícolas, ganaderos y urbanos (CastañoMeneses y Palacios-Vargas, 2003).

La urbanización es una de las grandes amenazas para la perdida de la diversidad, la conversión de ambientes naturales en zonas urbanas, áreas comerciales, parques industriales e infraestructura asociada es aproximadamente el $4 \%$ de la superficie de los continentes resultando en fragmentación y pérdida de hábitat (Lutinski et al., 2013). Los ecosistemas urbanos son áreas sometidas a constantes cambios por las actividades humanas, caracterizados por heterogeneidad espacial y dinámica temporal, lo que se traduce en desorden y reducción de biodiversidad (de Souza et al., 2012). En los bosques conservados se encuentra un mayor número de especies que en lotes baldíos en los jardines urbanos; también en áreas con vegetación nativa se tienen más especies que en cultivos, por la complejidad de la vegetación y las oportunidades como nuevos ecosistemas, pero las zonas de transición o de borde llegan a ser altamente diversas (Rodríguez-de León et al., 2019; Uno et al., 2010).

Las especies más ampliamente dispersadas por el planeta y comunes en las zonas urbanas son denominadas introducidas, algunas presentan un comportamiento llegándose a nombrar invasoras y además se convierten en plaga, pues responden positivamente al efecto de urbanización. Ejemplos de especies introducidas y consideradas plaga, son: Anoplolepis gracilipes, conocida como plaga agrícola; Cardiocondyla emeryi, Linepithema humile, Monomorium pharaonis, 
Paratrechina longicornis, Tapinoma melanocephalum consideradas plagas urbanas; todas ellas se pueden encontrar en la zona metropolitana de Guadalajara (Toennisson et al., 2011; VásquezBolaños, 1998; Vásquez-Bolaños et al., 2019). Las hormigas urbanas han recibido poca atención por los especialistas, 3.6\% de los trabajos dedicados a hormigas, a pesar de las importantes implicaciones para entender la ecología de los sistemas urbanos por el papel de las hormigas en las ciudades; para México no es la excepción, hay pocos estudios sobre hormigas urbanas (Santos, 2016).

Para Jalisco se tienen pocos trabajos sobre hormigas, destacan una lista comentada para el estado (VásquezBolaños y Navarrete-Heredia, 2004); un inventario del estero El Salado en Puerto Vallarta (Vásquez-Bolaños et al., 2014); el estudio de la subfamilia Dorylinae en Jalisco (Alatorre Bracamontes, 2016) y la subfamilia Dorylinae en Chamela (Watkins, 1988).

Con el objetivo de conocer qué especies de hormigas se encuentran en el Centro Universitario de Ciencias Biológicas y Agropecuarias de la Universidad de Guadalajara se estudió la mirmecofauna del lugar.

\section{Materiales y Métodos}

El Centro Universitario de Ciencias Biológicas y Agropecuarias se localiza en el municipio de Zapopan, a una altitud promedio de $1660 \mathrm{msnm}$, con vegetación secundaria y cultivos experimentales. Se colectaron hormigas de manera directa desde junio del año 2011 y hasta febrero del 2020, se revisaron jardineras, arboles y troncos en busca de hormigas, así como áreas de cultivo. Las hormigas se conservaron en alcohol al 70\%, se separaron y determinaron con las claves especializadas para el grupo; se etiquetaron con la información de colecta: país, estado, municipio, localidad, sitio, coordenadas, altitud sobre el nivel del mar, tipo de vegetación, fecha de colecta y colector o colectores; además de la información taxonómica: especie (o género en algunos casos) descriptor y fecha de descripción, y determinador. El material está depositado en la Colección Entomológica del Centro de Estudios en Zoología de la Universidad de Guadalajara. Se elaboró una lista de las especies colectadas destacando las especies introducidas.

\section{Resultados y Discusión}

Se tienen 39 especies de hormigas, agrupadas en 22 géneros $y$ seis subfamilias, para el Centro Universitario de Ciencias Biológicas y Agropecuarias, eso representa el $24 \%$ de lo que se conoce para el estado de Jalisco (VásquezBolaños, 2020). Se logró determinar a nivel especie el $80 \%$ del material, no fue posible determinar el $20 \%$ restante debido a la complejidad del género o por la falta de claves taxonómicas. La subfamilia Myrmicinae es la más diversa, está representada por 15 especies y nueve géneros; le sigue Dorylinae con diez especies y tres géneros; Formicinae con siete especies y cinco géneros; Dolichoderinae con tres especies y tres géneros; Pseudomyrmecinae con tres especies y un género; Ponerinae con una especie y un género.

Los géneros con mayor número de especies son Neivamyrmex, Pheidole y Pseudomyrmex, con ocho, cuatro y tres especies respectivamente. Cinco géneros están representados por dos especies cada uno y 14 géneros con una especie. 
De acuerdo a la literatura nueve especies son introducidas: Anoplolepis gracilipes, originaria de Asia; Cardiocondyla emeryi, originaria de África; Cardiocondyla minutior, originaria de Asia; Hypoponera punctatisima, originaria de África;
Linepithema humile, originaria de América del Sur; Monomorium pharaonis, originaria de África; Paratrechina longicornis, originaria de Asia; Tapinoma melanocephalum originaria de Asia y Tetramorium simillimum, originaria de África.

Lista de especies de hormigas presentes en el CUCBA. *Especies introducidas.

Formicidae Latreille, 1809

Dolichoderinae Forel, 1878

Leptomyrmecini Emery, 1913

Dorymyrmex Mayr, 1866

Dorymyrmex insanus (Buckley, 1866)

Linepithema Mayr, 1866

Linepithema humile (Mayr, 1868)*

Tapinomini Emery, 1913

Tapinoma Föerster, 1850

Tapinoma melanocephalum (Fabricius, 1793)*

Formicinae Latreille, 1809

Camponotini Forel, 1878

Camponotus Mayr, 1861

Camponotus atriceps (Smith, 1858)

Camponotus mina Forel, 1879

Lasini Ashmead, 1905

Anoplolepis Sanschi, 1914

Anoplolepis gracilipes (Smith, 1857)*

Plagiolepidini Forel, 1886

Brachymyrmex Mayr, 1868

Brachymyrmex heeri Forel, 1874

Brachymyrmex sp.

Nylanderia Emery, 1906

Nylanderia sp.

Paratrechina Motschoulsky, 1863

Paratrechina longicornis (Latreille, 1802)*

Pseudomyrmecinae Smith, 1952

Pseudomyrmecini Smith, 1952

Pseudomyrmex Lund, 1831

Pseudomyrmex championi (Forel, 1899)

Pseudomyrmex gracilis (Fabricius, 1804)

Pseudomyrmex pallidus (Smith, 1855)

Dorylinae Leach, 1815

Dorylini Leach, 1815

Labidus Jurine, 1807 
Labidus coecus (Latreile, 1802)

Neivamyrmex Borgmeier, 1940

Neivamyrmex andrei (Emery, 1901)

Neivamyrmex cornutus Watkins, 1975

Neivamyrmex harrisii (Haldeman, 1852)

Neivamyrmex melanocephalus (Emery, 1895)

Neivamyrmex nigrescens (Cresson, 1872)

Neivamyrmex sumichrasti (Norton, 1868)

Neivamyrmex swainsonii (Shuckard, 1840)

Neivamyrmex texanus Watkins, 1972

Nomamyrmex Borgmeier, 1936

Nomamyrmex esenbeckii (Westwood, 1842)

Ponerinae Lepeletier de Saint-Fargeau, 1835

Ponerini Lepeletier de Saint-Fargeau, 1835

Hypoponera Santschi, 1938

Hypoponera punctatissima (Roger, 1859)*

Myrmicinae Lepeletier de Saint-Fargeau, 1835

Attini Smith, 1858

Atta Fabricius, 1818

Atta mexicana (Smith, 1858)

Pheidole Westwood, 1839

Pheidole hirtula Forel, 1899

Pheidole tepicana Pergande, 1896

Pheidole sp. 1

Pheidole sp. 2

Trachymyrmex Forel, 1893

Trachymyrmex sp.

Crematogastrini Forel, 1893

Cardiocondyla Emery, 1869

Cardiocondyla emeryi Forel, 1881*

Cardiocondyla minutior (Forel, 1899*

Crematogaster Lund, 1831

Crematogaster opaca Mayr, 1870

Crematogaster sp.

Tetramorium Mayr, 1855

Tetramorium simillimum (Smith, 1851)*

Pogonomyrmecini Ward, Brady, Fisher \& Schultz, 2014

Pogonomyrmex Mayr, 1868

Pogonomyrmex wheeleri Olsen, 1934

Solenopsidini Forel, 1893

Monomorium Mayr, 1855

Monomorium minimum (Buckley, 1867)

Monomorium pharaonis (Linnaeus, 1758)*

Solenopsis Westwood, 1840

Solenopsis geminata (Fabricius, 1804) 


\section{Conclusiones}

Las hormigas son organismos que fácilmente pueden ser transportados por el hombre de manera accidental, se adaptan rápido a los ambientes a los que llegan y se asienten sin mayor problema. Las zonas que han sido fragmentadas por la creciente población humana y sus necesidades de urbanización, agricultura y ganadería; también llamadas zonas de ecotono o de borde, concentran una alta riqueza de especies de hormigas, pues coexisten tanto las especies nativas como las introducidas que son favorecidas con las condiciones del nuevo ambiente. Se tiene una gran cantidad de especies de hormigas en el área del CUCBA, considerando que para el estado de Jalisco se conocen 161 especies y un alto porcentaje de hormigas son especies introducidas, casi una cuarta parte. Para las especies introducidas no se conoce el impacto que causan hacia las especies nativas ni el impacto en el ecosistema, pues no se han realizado trabajos que documenten su actividad distribución $\mathrm{y}$ comportamiento.

\section{Literatura citada}

Alatorre Bracamontes, C. E. 2016. Taxonomía y distribución de las hormigas legionarias (Hymenoptera: Formicidae: Dorylinae) en el estado de Jalisco, México. Tesis de maestría, Maestría en Ciencias en Biosistemática y Manejo de Recursos Naturales y Agrícolas, Universidad de Guadalajara.

Arcila, A.M. y F.H. Lozano-Zambrano. (2003). Hormigas como herramienta para la bioindicación y el monitoreo. pp. 159-166. En: Fernández, F. (ed.). Introducción a las hormigas de la región neotropical. Instituto de Investigación de Recursos Biológicos
Alexander von Humboldt, Bogotá, Colombia. 424 pp. [ISBN 958-815123-6]

Castaño-Meneses, G. y J.G. PalaciosVargas (2003). Effects of fire and agricultural practices on neotropical ant communities. Biodiversity and Conservation, 12, 1913-1919.

Chacón de Ulloa, P. (2003). Hormigas urbanas. pp. 351-363. En: Fernández, F. (ed.). Introducción a las hormigas de la región neotropical. Instituto de Investigación de Recursos Biológicos Alexander von Humboldt, Bogotá, Colombia. 424 pp. [ISBN 958-815123-6]

Dáttilo, W., M. Vásquez-Bolaños, D.A. Ahuatzin, R. Antoniazzi, E. ChávezGonzález, E. Corro, P. Luna, R. Guevara, F. Villalobos, R. MadrigalChavero, J.C. De Faria Falcão, A. Bonilla-Ramírez, A.R. García Romero, A. De La Mora, A. RamírezHernández, A.L. Escalante-Jiménez, A.P. Martínez-Falcón, A.I. Villarreal, A. García Colón Sandoval, B. Aponte, B. Juárez-Juárez, C. CastilloGuevara, C.E. Moreno, C. Albor, D.L. Martínez-Tlapa, E. HuberSannwald, F. Escobar, F.J. MontielReyes, F. Varela-Hernández, G. Castaño-Meneses, G. Pérez-Lachaud, G.R. Pérez-Toledo, I. AlcaláMartínez, I.S. Rivera-Salinas, I. Chairez-Hernández, I.A. ChamorroFlorescano, J. Hernández-Flores, J. Martínez Toledo, J.-P. Lachaud, J.L. Reyes-Muñoz, J.E. ValenzuelaGonzález, J.V. Horta-Vega, J.D. Cruz-Labana, J.J. Reynoso-Campos, J.L. Navarrete-Heredia, J.A. Rodríguez-Garza, J.F. PérezDomínguez, J. Benítez-Malvido, K.K. Ennis, L. Sáenz, L.A. Díaz-Montiel, L.A. Tarango-Arámbula, L.N. Quiroz-Robedo, M. Rosas-Mejía, M. Villalvazo-Palacios, M. Gómez- 
Lazaga, M. Cuautle, M.J. AguilarMéndez, M.L. Baena, M. MadoraAstudillo, M. Rocha-Ortega, M. Pale, M.A. García-Martínez, M.A. SotoCárdenas, M.M. Correa-Ramírez, M. Janda, P. Rojas, R. Torres-Ricario, R.W. Jones, R. Coates, S.L. GómezAcevedo, S. Ugalde-Lezama, S.M. Philpott, T. Joaqui, T. Marques, V. Zamora-Gutierrez, V. Martínez Mandujano, Z. Hajian-Forooshani e I. Macgregor-Fors. (2020). Mexico ants: incidence and abundance along the Neartic-Neotropial interface. Ecology, 1-65. Doi: 10.1002/ECY.2944

De Souza, D. R., S. G. dos Santos, C. de B. Munhae y M. S. de C. Morini. (2012). Diversity of epigeal ants (Hymenoptera: Formicidae) in urban áreas of Alto Tietê. Sociobiology, 59 (3), 703-717.

Lutinski, J.A., B. Cortes Lopes y A.B. Barros de Morais. (2013). Diversidade de formigas urbanas (Hymenoptera: Formicide) de dez cidades do sul do Brasil. Biota Neotropical, 13(3), 332-342.

Rodríguez-de León, I.R., C.S. VenegasBarrera, M. Vásquez-Bolaños y J.V. Horta-Vega. (2019). Estructura de la comunidad de Formicidae (Hymenoptera) en dos agroecosistemas con diferente grado de perturbación. Agrociencia, 53(2), 285-301.

Santos, M. N. (2016). Research on urban ants: approaches and gaps. Inectes Sociaux, 63, 359-371. Doi 10.1007/s00040-016-0483-1

Toennisson, T.A., y N.J. Sandres, W.E. Klingeman y K. M. Vail. (2011). Influences on the structure of suburban ant (Hymenoptera: Formicidae) communities and the abundance of Tapinoma sessile.
Community and Ecosystem Ecology, 40(6), 1397-1404.

Uno, S., J. Cotton y S.M. Philpott. (2010).

Diversity, abundance, and species composition of ants in urban green spaces. Urban Ecosystems, 13, 425441.

Vásquez-Bolaños, M. M. (1998). Anoplolepis longipes Jerdon, 1852 (Hymenoptera: Formicidae). Dugesiana, 5(1), 44-45.

Vásquez-Bolaños, M. (2015). Taxonomía de Formicidae (Hymenoptera) para México. Métodos en Ecología y Sistemática, 10(1), 1-55.

Vásquez-Bolaños, (2020). Hormigas de México. Lista de las especies de hormigas de México: Jalisco. https://sites.google.com/udg.mx/horm igasdemexico/hormigas-dem\%C3\%A9xico/jalisco. Consultado el 12de febrero de 2020.

Vásquez-Bolaños, M., A. CisnerosCaballero y J. L. Navarrete-Heredia. (2014). Hormigas. pp. 113-120. En: Guerrero, S., J. L. Navarrete-Heredia y S. H. Contreras-Rodríguez (Coordinadores). Biodiversidad del Estero El Salado. Universidad de Guadalajara, Jalisco, México. 219 pp. [ISBN 978-607-7420-4]

Vásquez-Bolaños, M., A.L. GonzálezHernández y G.A. Quiroz-Rocha. (2019). Hormigas (Hymenoptera: Formicdae) del Bosque Los Colomos, Guadalajara, Jalisco, México. Digital Ciencia@UAQRO, 12(2), 23-27.

Vásquez-Bolaños, M. y J.L. NavarreteHeredia. (2004). Checklist of the ants (Hymenoptera: Formicidae) from Jalisco, México. Sociobiology, 43(2), 351-365.

Watkins, J.F. II. (1988). The army ants (Formicidae: Ecitoninae) of the Chamela Biological station in Jalisco, Mexico. Folia Entomológica Mexicana, 77, 379-393. 